work on interchange reactions of this type, to treat this aspect quantitatively.

Our results indicate, however, that there is a parallelism between isotopic interchange and isomerisation. Of the various mechanisms which have been proposed for three-carbon tautomerism, those representing the change as purely intramolecular appear to be unacceptable. Further discrimination between intermolecular mechanisms must await evaluation of the equilibrium constant of the exchange reaction; this work is actively in hand.

Birkbeck College, London, E.C.4. D. J. G. Ives.

Imperial College, London, S.W.7. Aug. 21.

\section{Fertilisation of Successive Broods of Gammarus chevreuxi}

IN a letter in NATuRE of May 18, p. 832, an account was given by $\mathrm{Mr}$. K. W. Yarnold of some breeding experiments with red-eyed animals of a new stock (1933) of Gammarus chevreuxi, Sexton, crossed with the red-eyed Stock II (1922). Both these stocks originated in the Marine Biological Laboratory, and have always given red-eyed young when bred inter se, or when crossed with each other.

Mr. Yarnold, however, records an instance of a single black-eyed appearing in the offspring from a mating of two red-eyed (new red $\times$ Stock II red), the female of which had been previously mated with a black-eyed male. His explanation is "that some of the sperms from the previous mate of the female had remained behind and fertilised one or more of the eggs, causing the appearance of a heterozygous black-eyed specimen-not an unusual phenomenon in Gammarus".

It seems to me that if this explanation were correct, the parentage of the offspring from any cross-mating would always be in doubt, and the genetical work done on Gammarus chevreuxi would have no value. In my own experience-and I have worked on this species for twenty-three years-I have never had a single instance of this carrying over of sperm from one mating to the next in the many hundreds of similar cross-matings made, and I think that a study of the structure of the animals and of their mating habits proves conclusively that such an occurrence is impossible.

The female Gammarus chevreuxi has an external incubatory pouch made up of four pairs of broodplates or lamellæ attached to the ventral surface of the 2nd, 3rd, 4th and 5th peræon-segments. The oviducts, one each side, open into the pouch on the ventral surface of the 5th segment. The pouch is held together when in use by long flexible hairs, and is open at both ends, where the lamellæ are separated by the width of the body. A constant steady stream of water is beaten through it from behind forwards by the pleopods, which serves to keep the eggs aerated and to remove all extraneous matter, for example, eggs which are not fertilised macerate, and are swept away by this current.

The cuticle, of hard unyielding chitin, is continuous over the entire body surface. This hard skin has to be sloughed off before the female can mate, and mating can only take place directly after the moult while the new skin is soft and elastic enough to permit of the passage of the eggs through the oviduct apertures into the pouch.
In moulting, the cuticle cracks across the dorsum just behind and around the head, and the animal heaves itself up and draws out backwards, leaving the old skin unbroken on the ventral surface. The moults are always perfect to the last detail in healthy specimens but, unless carefully watched for, they are very rarely seen in this condition owing to the habit amphipods have of devouring their cast skins immediately after ecdysis.

Sickly animals have great difficulty in moulting, and an incomplete or imperfect moult always ends in death, but sickly animals do not breed. I have never seen a healthy Gammarus pair with an unhealthy one ; it may take hold of it, but only for the purpose of killing and eating it, never for breeding.

Fertilisation takes place outside the body. The male, which has paired with and carried the female for some days, assists her in moulting, and then it ejects the sperm into the open pouch. The female commences to extrude the eggs, and as she does so, she breaks free and resists any further mating. No pairing, even, takes place until she is once more in the right physiological condition, and no mating again without a complete moult.

We have made it a rule here, in all cross-matings, after the females moult, to give the pair a fresh bowl, water and food, so as to avoid any confusion with the offspring of a previous mating. The young are semi-transparent and only a millimetre in length on hatching, and as their instinct is to keep out of the light, it is sometimes very difficult to find them among the debris in the bowl.

Taking these facts into consideration: $(a)$ that the animals never pair until the female is in the right physiological condition, and that the female will resist being taken unless in this condition; $(b)$ that, once paired, they remain together until mating is over; (c) that mating must always be preceded by a moult; (d) that the animal in moulting comes out of the old skin, on the dorsal surface, leaving the ventral surface unbroken; $(e)$ that the old pouch is sloughed off as a whole (with any young that may still be in it); $(f)$ that fertilisation takes place in the open pouch; $(g)$ that the sperm of the new mating is ejected into the new pouch; and $(h)$ that a steady current of water is driven through the pouch from end to end during the whole period of incubation, I think it is evident that some other explanation than the one given by $\mathrm{Mr}$. Yarnold for the appearance of a black-eyed young in his red-eyed cultures must be looked for.

\section{Marine Biological Laboratory,}

$$
\text { E. W. Sexton. }
$$
Plymouth. Aug. 16.

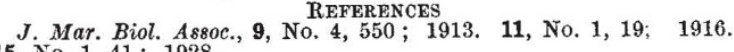
15 , No. 1,$41 ; 1928$

\section{Distribution of Nematodes in the Small Intestine of the Sheep}

In the course of ecological studies on the nematodes of the small intestine of sheep, particularly during their parasitic existence, it has become apparent that the manner of their distribution throws light upon the relation between host and parasite.

The jejenum is the favoured region of infection of species naturally occurring in sheep; those of any one genus share a common area, while species of different genera vary in the degree to which their infections overlap. 